\title{
Chemie in unserer Zeit
}

\section{Eugens scibold Die Suche nach Rohstoffen}

Abb. 1. Plattengrenzen, Auseinanderdriften der Ozeanböden und Lagerstätten. An divergierenden Plattenrändern (rote Linien) kommt es durch Zufuhr aus dem Erdmantel und Wechselwirkung mit dem in die aufsteigenden Basalte eindringenden Meerwasser zur Fällung von Metallhydroxiden und -sulfiden (schwarze Punkte). Dazu gehören auch die Erzschlamme im Roten Meer. An konvergierenden Rändern (blaue Linien) taucht die ozeanseitige Platte $a b$, wodurch Gesteine in der Tiefe umgeschmolzen und mobilisiert werden. Dadurch können Erzlagerstätten entstehen, wie die Kupfer/Molybdän-Vorkommen am Westrand von Süd- und Nord-Amerika. Neue ozeanische Kruste entsteht an den divergierenden Rändern. Sie driftet nach beiden Seiten auseinander, ist also um so älter, je weiter sie von diesen Rändern entfernt ist. Das zeigt das Krustenalter auf der Karte: $\mathrm{T}=$ Tertiär (bis vor 65 Millionen Jahren), $\mathrm{K}=\mathrm{Kreide}$ (bis vor 136 Millionen Jahren), $\mathrm{J}=$ Jura (bis vor 190 Millionen Jahren). Während des Auseinanderdriftens lagern sich auf der Kruste Sedimente ab. Die Sedimentmächtigkeiten sind daher auf der jüngeren Kruste geringer als auf der - landnäheren - älteren. Dort kommt die erhöhte Anlieferung von Material aus den Kontinenten hinzu. Dies ist wichtig für die Lagerstätten von Kohlenwasserstoffen an Kontinentalrändern (vgl. Abbildung 10b). Nach [10]

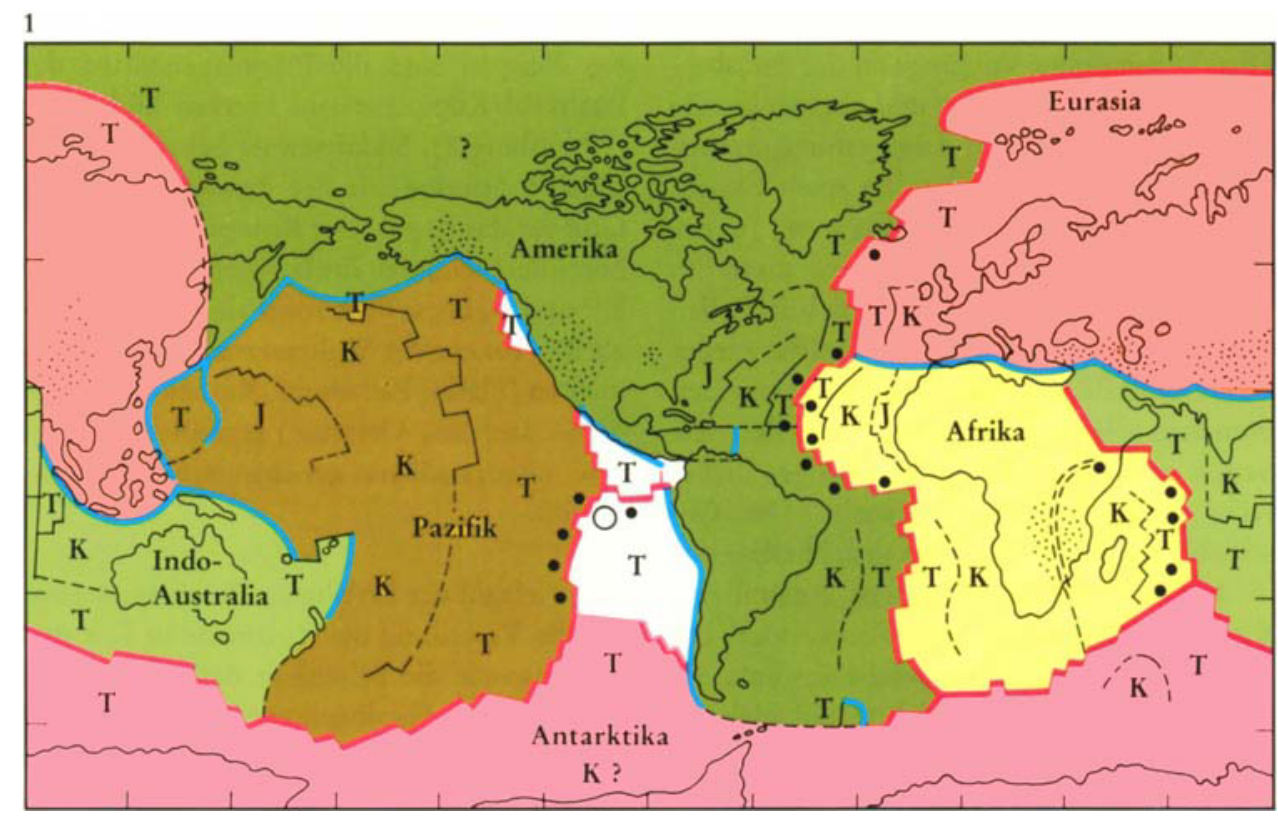

Wenn ein Geologe über die Suche nach Rohstoffen nachdenkt, wird er sich daran erinnern, daß diese Suche die Wurzel seiner Wissenschaft war, bei der also die Anwendung von Anfang an mit im Blickfeld lag. Er sieht den Einfluß der Rohstoffe auf die Kultur durch die Jahrtausende an der Gliederung unserer Geschichte in Steinzeit - Bronzezeit - Eisenzeit und fragt sich, ob man später von unseren Tagen als dem Ubergang in die Polymerzeit sprechen wird. Das wird von den Rohstoffen und von den Chemikern abhängen, die darin erfahren sind, rohe Stoffe zu veredeln - nicht nur beim Wein.

Man nennt nicht alle geochemischen Konzentrationen von Elementen oder Verbindungen in oder auf unserer Erdkruste Rohstoffe. Diese Konzentrationen müssen mit Gewinn ausgebeutet werden können, was derzeit vielfach erst bei einer Konzentrierung auf das einige Tausendfache der Erdkrustenhäufigkeit möglich ist. Die mittlere Krustenhäufigkeit von Blei ist beispielsweise $12 \mathrm{~g}$ pro Tonne (ppm). Der "cut-off-grade" dafür, d.h. die Grenze der Bauwürdigkeit, lag 1975 bei $40000 \mathrm{~g}$ pro Tonne. Der Anreicherungs- faktor für eine wirtschaftliche Gewinnung war also bei Blei 3300 - bei Chrom ist er 2100, bei Zinn 2000 und bei Gold nur 1000, da dieses Metall so teuer ist und daher - bei einer Krustenhäufigkeit von nur 0,0035 ppm - die Grenze für einen wirtschaftlichen Abbau bei 3,5 ppm liegt [1]. Die Grenze der Bauwürdigkeit hängt natürlich von vielen Faktoren $a b$, die nichts mit der Geologie zu tun haben und auf die hier nicht näher eingegangen werden soll: Marktlage, Energiekosten, Gesetzgebung von der Prospektion bis zum Umweltschutz, Verkehrs- oder Arbeitsbedingungen.

\section{Grundsätzliches}

Die Rohstoffsuche beginnt also damit, daß man versucht, die Prozesse zu erkennen, die zu einer natürlichen Anreicherung der Elemente führen. Sie sind sehr zahlreich. Endogene, vom Erdinnern ausgehende Prozesse gehören dazu, die Magmen und Laven schaffen und bewegen, was zu Lagerstätten von Mineralen oder Erzen führen kann. Aber auch exogene, d.h. im wesentlichen von der Sonne gesteuerte Prozesse schaffen biologi- 
sche Rohstoffe wie Torf und Kohle, Erdöl und Erdgas. Sie sammeln Grundwasser und bilden Böden, beides Rohstoffe, von denen hier nicht die Rede sein soll. Exogene Prozesse tragen durch Wasser, Eis und Wind Gesteine ab und legen Tieferes, etwa Erzkörper, frei. Sie können Wertvolles wie Diamanten oder Gold in Fluß-Seifen oder Erze durch Verwitterung anreichern; sie können diese Rohstoffe aber auch verteilen und wieder verdünnen.

Die Zeugen dieser Vorgänge an der Erdoberfläche können überdeckt und abgesenkt, die abgelagerten Schichten dabei erhitzt, verbogen oder zerhackt werden. So spielt Exogenes und Endogenes zusammen, wenn aus organischem Material Oll wird, wenn dieses im Untergrund wandert, wenn es sich in Fallen anreichert und im günstigen Fall nicht wieder an die Oberfläche entweicht. Im allgemeinen brauchen alle diese Prozesse lange Zeit. Sie hatten zudem im Laufe der Erdgeschichte recht unterschiedliches Gewicht. Das Geschichtliche in der Geologie drückt sich etwa in den riesigen Eisenerzlagern Australiens, Kanadas, Venezuelas, Brasiliens oder der Ukraine aus, die alle das gleiche absolute Alter um zwei Milliarden Jahre und gleichen Charakter haben. Beides deutet darauf hin, daß sich der durch Pflanzen zunehmend erzeugte Sauerstoff zunächst nur im Meer anreicherte. Als seine Konzentration auch in der Atmosphäre merklich anstieg, wurde diese einzigartige metallogenetische („metallbildende") Epoche beendet. Am anderen Ende der Zeitskala steht der Mensch, der erst seit einem Jahrhundert zu einem auch geologisch wirksamen Faktor geworden ist.

Alle diese Prozesse haben und hatten regional unterschiedliches Gewicht. Das Klima bestimmt, ob sich Kohle oder Salze bilden, die ozeanische Zirkulation, ob Phosphorite oder Erdölmuttergesteine entstehen. Erzprovinzen können strukturell bestimmt sein: An den $70000 \mathrm{~km}$ langen sogenannten divergierenden Plattengrenzen wie auf den mittelozeanischen Rücken ist und war die Vererzung anders als an konvergierenden wie in der Umrandung des Pazifischen Ozeans (Abbildung 1). Ein Ausgangspunkt für diese Erkenntnisse war das Rote Meer. Im „AtlantisII-Loch“, das auf einem Divergenzrand liegt, wurden 1964/65 Metallanreicherungen entdeckt (Abbildung 2). Dies regte weitere weltweite Untersuchungen auf den mittelozeanischen Rücken an [2]. Es entstehen also in der Mitte oder an den Rändern von Ozeanen ver- schiedene metallogenetische Provinzen oder in den Kontinenten oder an deren Rändern auch verschiedene Ölprovinzen mit jeweils verwandten Zügen. Bereits die Vielzahl der genannten Faktoren, die ja auch noch in Zeit und Raum zusammenspielen müssen, um zu einer Anreicherung zu führen, erklärt die ungleiche Verteilung und die Seltenheit von Lagerstätten. Sie kommen sozusagen nur durch Zufälle zustande und sind im einzelnen leider meist relativ kleine oder dünne Körper.

Ein Beispiel sind die Platinlagerstätten des Bushveld-Komplexes im Herzen Südafrikas (Abbildung 3). Südafrika ist bekanntlich für Metalle dasselbe wie der Persisch/Arabische Golf für das Erdöl. Der Komplex enthält drei besonders wichtige Erzhorizonte (Abbildung 3, 4 und 5). Diese Horizonte allein sollen mehr als 80 Prozent der Weltreserven an Platinelementen (Platin, Palladium, Ruthenium, Rhodium, Iridium, Osmium) enthalten. Das ist eine unvorstellbare geochemische Konzentration.

Die Vielzahl der beteiligten Prozesse, die ungleiche Verteilung der Rohstoffe in Zeit und Raum sowie die Seltenheit der Lagerstätten haben für den Geologen vor allem zwei Konsequenzen: Sie erschweren die Suche nach Lagerstätten und auch die Abschätzung von Vorräten. Diese beiden Punkte werden im folgenden näher erläutert. Dabei sollen aber nicht die spezielle deutsche Situation behandelt und nur gelegentlich rohstoffpolitische oder wirtschaftliche Aspekte gestreift werden, obwohl diese für viele Überlegungen natürlich äußerst wichtig sind. Zunächst: Wie sucht man Rohstoffe?

\section{Prospektion}

Seit uralten Zeiten und nach wie vor sucht man an der Erdoberfläche - und nach wie vor ist der Individualist im Feld wichtig der ja auch den Charakter des Berufsstandes der Geologen nachhaltig prägt. Ein Erzhorizont tritt zutage, ist Hammer, Hacke und Schaufel zugänglich (Abbildung 5). Im Sand und Geröll der Flïsse kommt die Schüttelpfanne dazu. Asphalt deutet auf $\mathrm{Ol}$ in der Tiefe (Abbildung 6). Noch immer ist das Glück der beste Prospektor, und noch immer dürften mehr Erzlagerstätten durch Zufall gefunden werden als durch Voraussagen.

Doch man hilft in der Geologie mehr und mehr dem Glück nach, indem man die Suche auf höffige Bereiche konzentriert: auf die
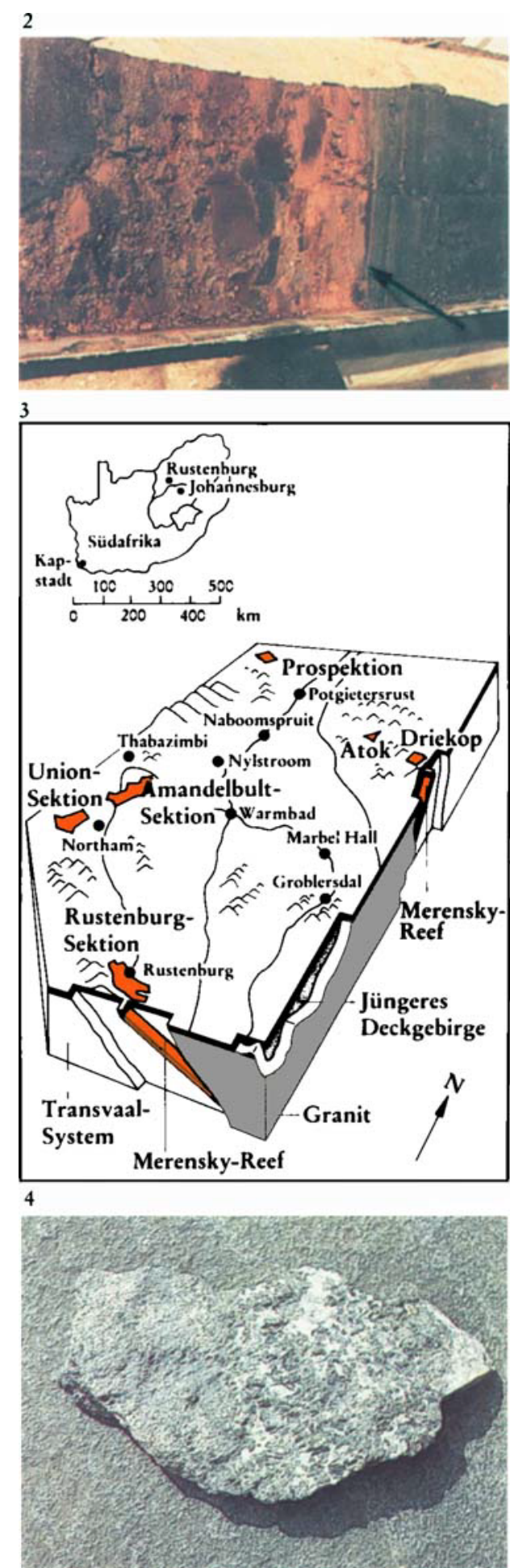

Ränder eines Granitmassivs, auf große Störungszonen, auf die strukturelle Fortsetzung bekannter Vorkommen. Die Basis dafür ist nach wie vor die geologische Karte. Wie viele Informationen sind für den Chemiker in einer Formel zusammengefaßt, etwa in $\mathrm{CH}_{3}-\mathrm{CH}_{2} \mathrm{OH}$ ! Die Formel des Geologen ist die Karte, die ihm Gesteine und deren Alter, Bild und Bau der Landschaft und des Untergrunds dreidimensional verrät, die ihm sogar ein vierdimensionales Bild gibt, das die Zeit 
Abb. 2. Kastenlotkern aus dem „AtlantisII-Loch“ im zentralen Roten Meer. Der Erzschlamm enthält in den dunklen Partien Mangan (bis über 30 Prozent) oder Sulfide (mit Gehalten an Kupfer bis 2 Prozent, an Zink bis 20 Prozent), in den rötlichen Partien Eisenhydroxide und -oxide. [Valdiviafahrt 3/1972; Photo: Fa. Preussag AG, Hannover]

Abb. 3. Merensky-Reef als Platin-Lagerstätte. Nordwestlich von Johannesburg liegt der Bushveld-Komplex aus Tiefengesteinen, die hauptsächlich vor 2100 Millionen Jahren aus dem Erdmantel durch Gesteine des Transvaal-Systems hochgedrungen sind. An der Oberfläche mißt der Komplex etwa $100 \mathrm{mal} 300 \mathrm{~km}$. Er enthält drei besonders wichtige Erzhorizonte, darunter das sogenannte Merensky-Reef. Es ist schüsselförmig gelagert und streicht auf rund $230 \mathrm{~km}$ an der Oberfläche aus. [Nach Rustenburg Platinum Mines Ltd.]

Abb. 4. Das Merensky-Reef ist zwischen 10 $\mathrm{cm}$ und $7,5 \mathrm{~m}$ mächtig - hier ein $40 \mathrm{~cm}$ mächtiges Handstück. Es besteht hauptsächlich aus dunklen Pyroxenen, hellen Plagioklasen und goldglänzenden Sulfiden, die im wesentlichen die Elemente der Platingruppe (Pt, Pd, Ru, Rh, Ir, Os) enthalten. Das Reef enthält fünf bis sechs Gramm pro Tonne von diesen Elementen.

Abb. 5. Suchgraben auf das an der Oberfläche ausstreichende Merensky-Reef. Unter einer dünnen Bodendecke steht das Reef an, das sich durch grinliche Verwitterungsfarben verrät. Im Hintergrund der Pilansberg (vgl. Abbildung 3).

Abb. 6. Zu den bekanntesten Oberflächenaustritten von Asphalt als Hinweis auf Erdöl im Untergrund gehört der Asphaltsee von Rancho La Brea in der Nähe von Los Angeles/Kalifornien. Das Vorkommen nimmt rund $70000 \mathrm{~m}^{2}$ ein und hat zahlreiche, bis ins Pleistozän reichende Tierreste konserviert.
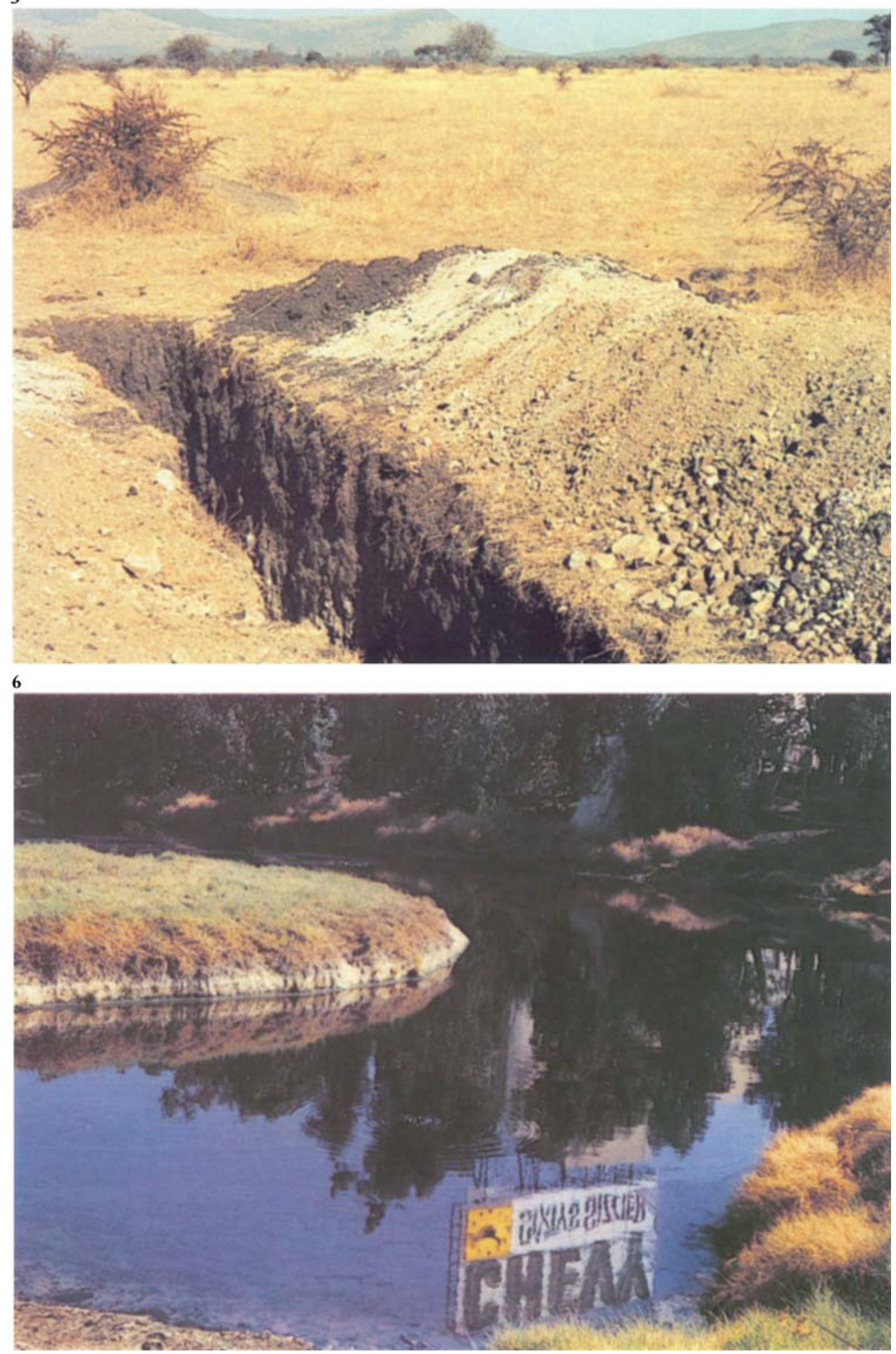

mit einschließt. Modernste Hilfsmittel, vor allem für schwer zugängliche Gebiete und für einen synoptischen Uberblick sind die Satelliten. Das "Remote Sensing" (etwa von Landsat ERTS $=$ Earth Resources Technology Satellite) enthüllt mit einer Auflösung von $80 \mathrm{~m}$ aus $910 \mathrm{~km}$ Höhe lineare oder kreisförmige Strukturen und, wenn man den Computer heranzieht, auch Gesteinstypen und Verwitterungsfarben, vor allem natürlich in ariden und daher vegetationsarmen Gebie- ten. Kupfervorkommen in Arizona und $\mathrm{Pa}$ kistan zum Beispiel wurden neuerdings auf diese Weise entdeckt. Vom Flugzeug aus können zusätzliche Methoden eingesetzt werden, vor allem aktive wie Untersuchungen mit Radar.

Weite Flächen der Erde aber sind von Wasser, Sümpfen, Wüstensand, tropischen Böden, glazialen Ablagerungen überdeckt, die den Untergrund verhüllen. Hier kann die geo- 


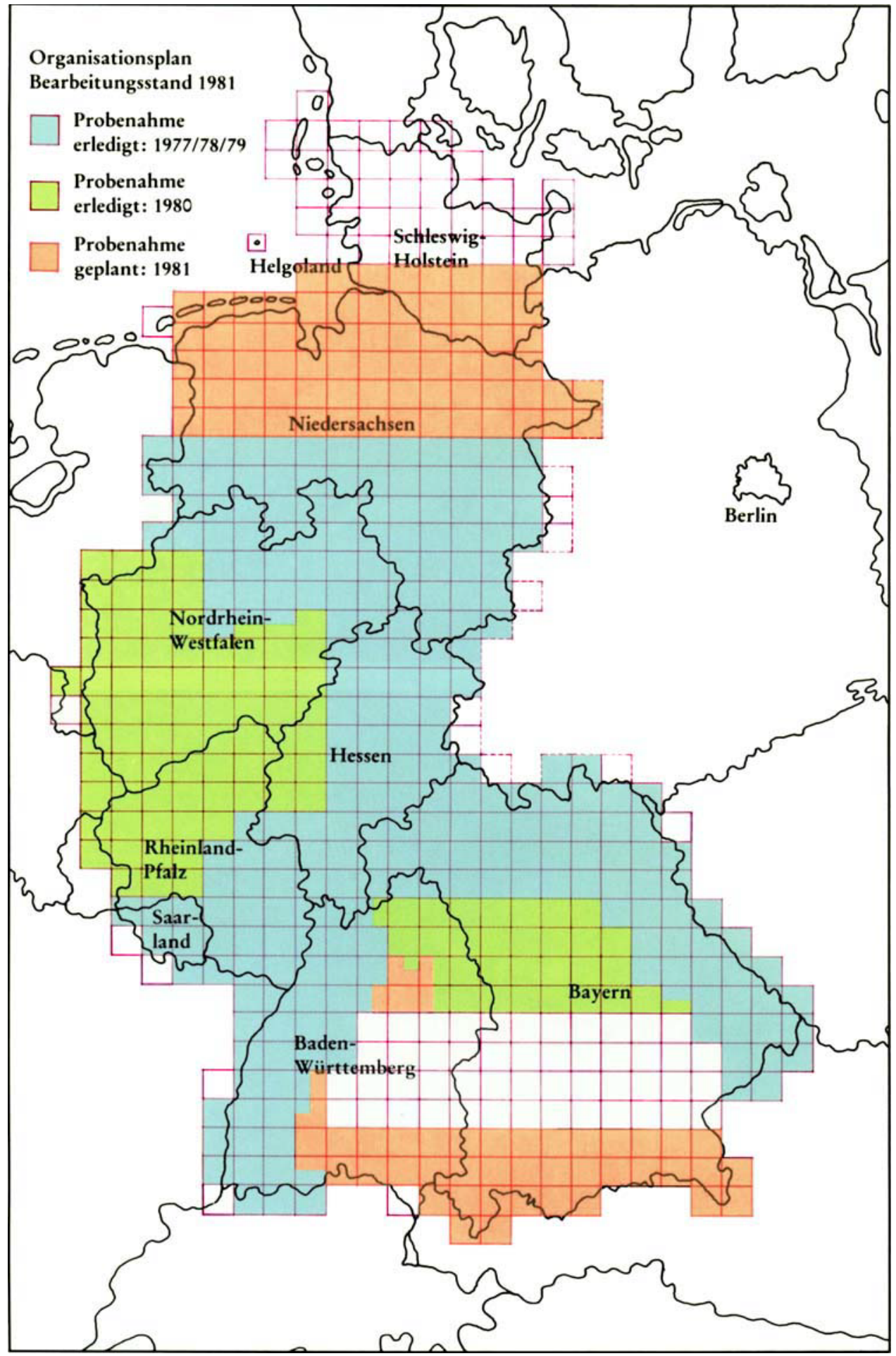

chemische Prospektion weiterhelfen. Anomalien im Untergrund können durch Grundwasser oder durch Pflanzen in die Böden hochprojiziert werden. Dabei sind die Elemente unterschiedlich mobil. In geochemischen "Halos" um Kupfervorkommen in Nevada wandern zum Beispiel Indium und Thallium am weitesten, sind also erste $\mathrm{Hin}^{-}$ weise für eine weitere Prospektion. Minerale können durch Wasser oder Eis verfrachtet werden. Sie werden teilweise gelöst. Ablage- rungen oder Wasser von Flüssen können also zu Erzkörpern in deren Einzugsgebiet führen. Seit 1977 wird in der „Bundesanstalt für Geowissenschaften und Rohstoffe" in Hannover an einem geochemischen Atlas der Bundesrepublik Deutschland gearbeitet, der $\mathrm{zu}$ einer Bestandsaufnahme - auch für Umweltfragen - in unserem Land führen soll (Abbildung 7).

Direkt ist der Untergrund aber nur durch
Abb. 7. Geochemischer Atlas der Bundesrepublik Deutschland. Seit 1977 analysiert die Bundesanstalt für Geowissenschaften und Rohstoffe, Hannover, 25 Elemente in möglichst unverschmutzten, natürlichen Oberflächenwässern aus Bächen und Quellen und entnimmt etwa alle zwei Quadratkilometer eine Probe. Anomalien können auf Erzvorkommen im Einzugsgebiet hinweisen, wenn Umwelteinfliusse ausgeschieden werden können. [Darstellung: Bundesanstalt für Geowissenschaften und Rohstoffe]

Abb. 8. a) Vereinfachte geologische Karte eines Ausschnitts von Südwestafrika [nach K. N. Downing und M. P. Coward, Geol. Rundschau, 70972 (1981)]. Die Gesteine und die von Nordosten nach Südwesten streichenden Strukturen des vor 750 und 520 Millionen Jahren entstandenen sogenannten Damara-Orogens werden im Südwesten an der Linie Walvis Bay - Gorob durch die jungen Wüstensedimente der Namib iiberdeckt. b) Eine aeromagnetische Aufnahme dieses Gebiets zeigt diese Strukturen auch unter der Namib. Sie biegen dort nach Südsüdwesten um. Diese detaillierten Ergebnisse stammen von einer Befliegung mit Profilabständen von einigen wenigen hundert Metern. [Photo: Dr. P. D. Toens, Atomic Energy Board, Südafrika]

teure Bohrungen zu erreichen. "The diamond drill is the most important secret for successful mine-hunters. " $:$ Trotzdem werden indirekte, geophysikalische Methoden immer wichtiger, auch wenn sie zunächst kein Probenmaterial für Analysen liefern. Sie

* "Der Diamantbohrer ist das wichtigste Geheimnis des erfolgreichen Jägers auf Lagerstätten“ (so hört man in Kanada). 


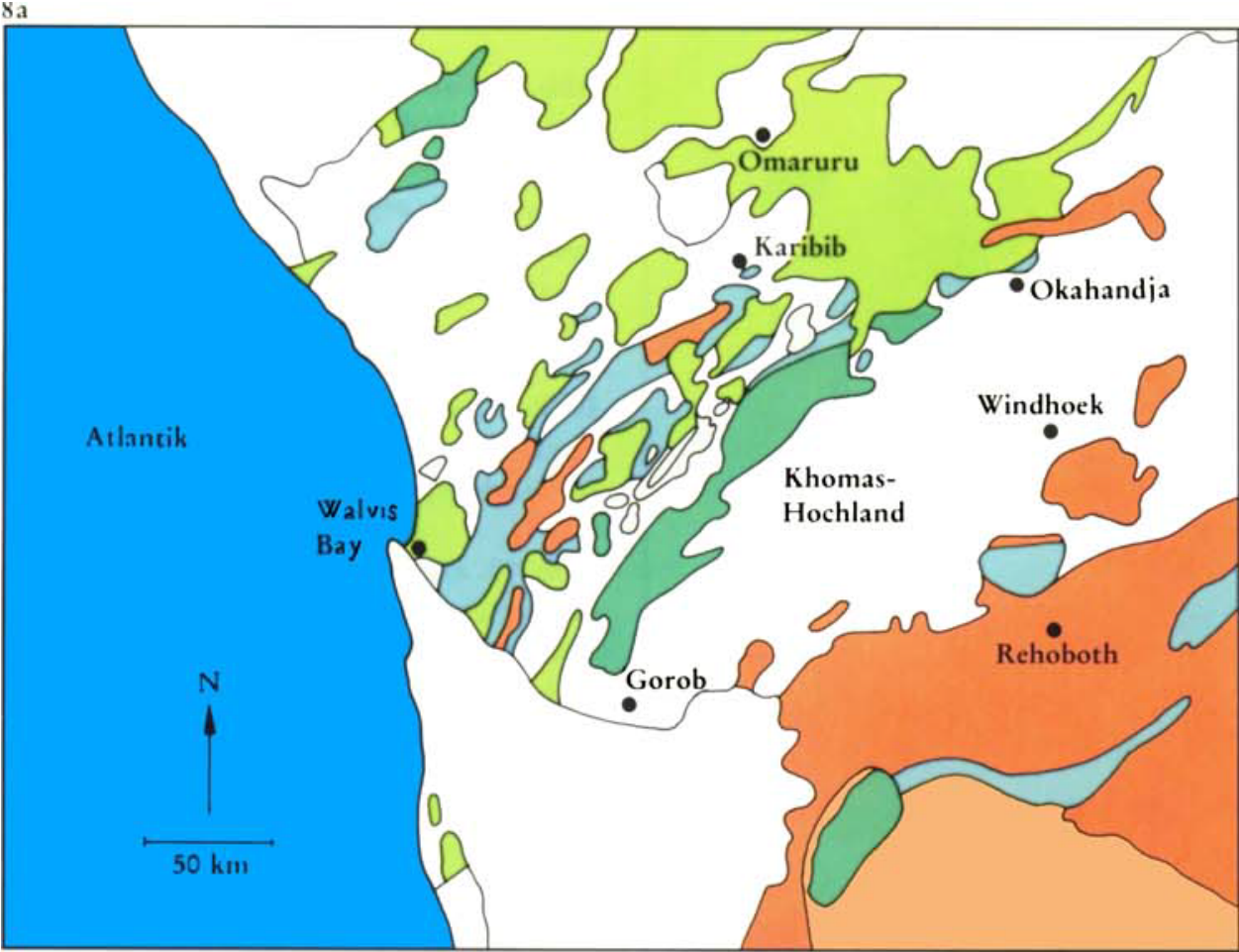

$8 b$

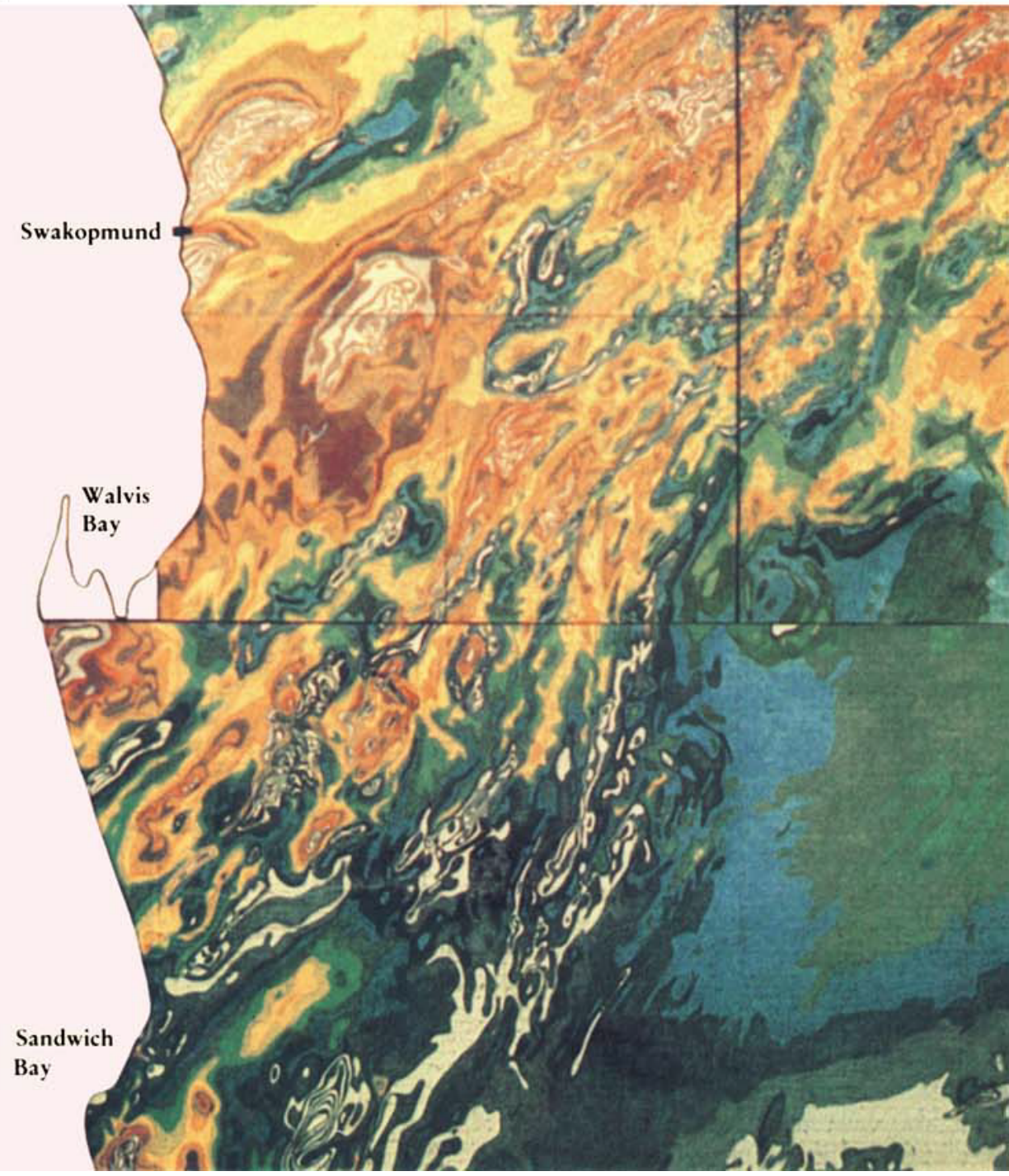

liefern aber selbst beim Grundeinsatz, mehr noch vom Schiff oder Flugzeug aus, einen raschen Uberblick für unbekannte und entlegene Gebiete, aber auch für die Neu-Interpretation bereits bekannter Bergwerksdistrikte. Man geht vor allem magnetischen (Abbildung 8), gravimetrischen oder sonstigen Anomalien nach, die man dann am Boden oder in Bohrungen näher untersucht. In $\mathrm{Ka}$ nada sind derzeit Magnetometer im Einsatz, die aus $150 \mathrm{~m}$ Höhe im Abstand von $300 \mathrm{~m}$ Profile messen. Ein Typ integriert bis in mehrere Kilometer Tiefe; ein anderer, der Gradienten mißt, erfaßt Anomalien des Grundgebirges bis zu einigen $100 \mathrm{~m}$ Tiefe unter einigen $10 \mathrm{~m}$ Uberdeckung. Daten zur Lagerung und sogar zum Charakter der Gesteine im tieferen Untergrund liefern seismische Verfahren. Ihre neuerdings dreidimensionale Anwendung und Auswertung mit Hilfe immer größerer Computer macht laufend Fortschritte, so daß es sogar gelungen ist, anhand solcher Daten Gas in Speichergesteinen $\mathrm{zu}$ identifizieren.

Die Zukunft liegt im Auffinden tieferer, entlegener und ärmerer Vorkommen. All das setzt voraus, daß diese Lagerstätten größer sein müssen, womit auch der Suchaufwand größer wird. Dasselbe gilt für die Suche nach komplexeren Vorkommen.

Für die Zukunft brauchen wir also verbesserte Prospektionsmethoden und neue Konzepte zur Entstehung, Verbreitung und Ausdehnung der Lagerstätten. Die Arbeiten an die sen Problemen sind zugleich typische Aufgaben der geologischen Grundlagenforschung.

Zum Beispiel entwickelten nach dem zweiten Weltkrieg Erdölgesellschaften in Zusammenarbeit mit Hochschulen zuerst an der Mündung von Rhône und Orinoco neue und genauere Vorstellungen über die Bildung und den Aufbau von Fluß-Deltas. Im Anschluß daran entwickelte sich die Olförderung im Niger-Delta explosiv. Grundlage war das alte Konzept, daß sich die geologischen Verhältnisse an Land über die Küste hinaus fortsetzen, was auch in vielen Fällen zutrifft. Allerdings hätte man mit dieser Vorstellung niemals vor dem Grundgebirge Norwegens in der Nordsee nach $\mathrm{O} l$ gesucht. Grundlage für die Suche an dieser Stelle ist seit 20 Jahren das neue Konzept der Ozeanentstehung. Untersuchungen in der Arabischen Wüste galten zunächst als akademische Delikatesse - bis offenkundig wurde, daß dahinter der Wunsch stand, das 250 Millionen Jahre alte 


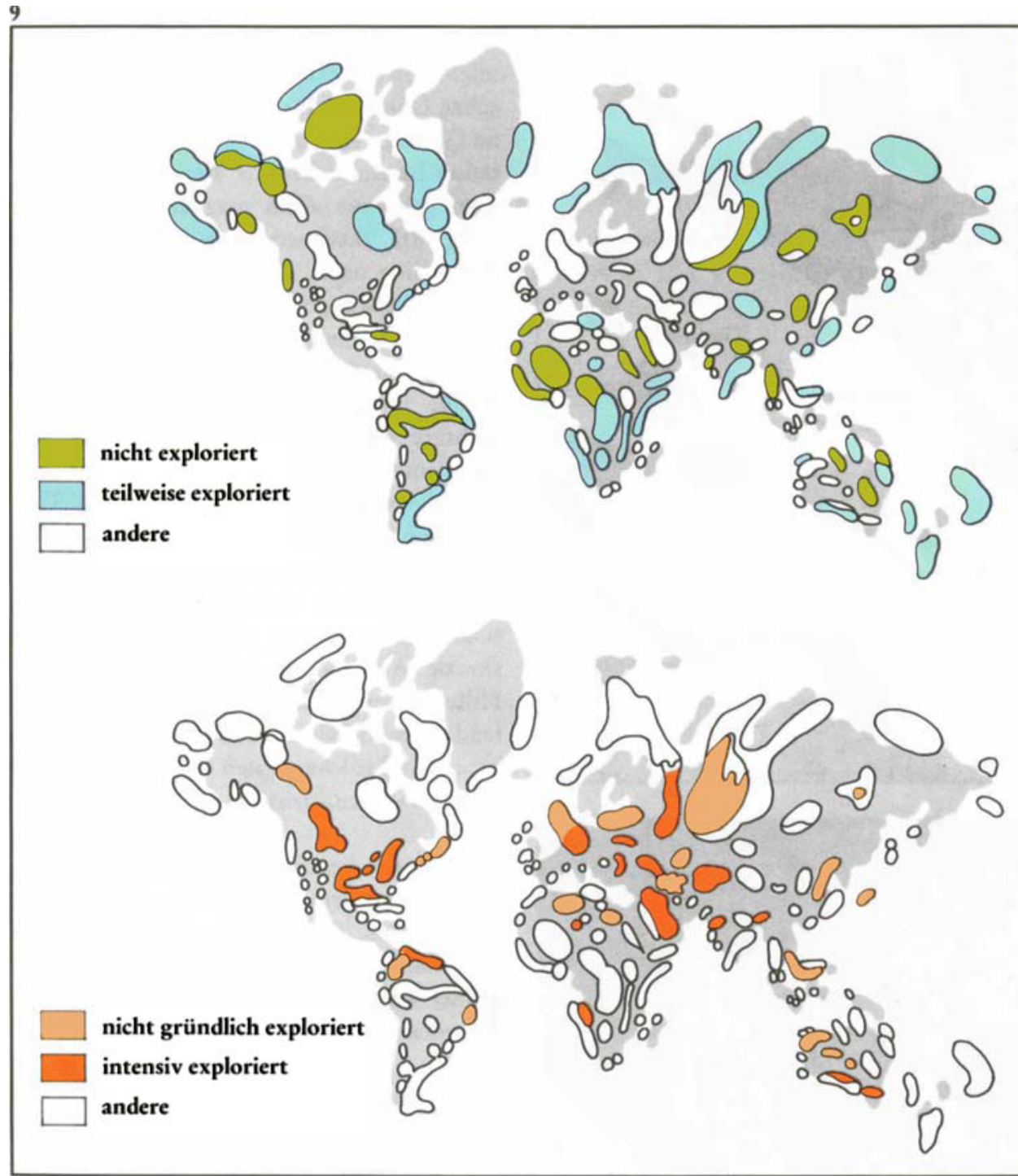

Speichergestein des Erdgases in der Nordsee besser verstehen zu lernen. Heute fragt man sich - immer noch akademisch - wie potentielle Mutter- und Speichergesteine in der Tiefsee entstehen, wie sie aussehen, wo sie vorkommen, zum Beispiel die Tiefsee-Sande und Carbonat-Körper aus abgesenkten fossilen Flachwasserablagerungen.

Die moderne Erdölexploration richtet sich nicht mehr in erster Linie auf potentielle Olfelder, sondern analysiert ganze Sedimentbecken, in denen sie auftreten können, ihre Struktur, ihre geschichtliche Entwicklung, die Gesteinsausbildung und -entstehung, die Wärmeströme sowie geochemische Korrelationen vom OIl und Gas bis zum Muttergestein. Solche Analysen werden heute durch elektronische Datenspeicherung und -verarbeitung wesentlich erleichtert. Die Übersichtsbegehung, Prospektion und die anschließende detaillierte Exploration werden damit allerdings leider auch immer teurer.
Abb. 9. Von den rund 600 Sedimentbecken der Erde sind bisher etwa 400 exploriert: bei 240 davon haben sich keine wirtschaftlichen Funde ergeben. Die Exploration von rund 160 Becken dagegen war auch wirtschaftlich erfolgreich. Freilich enthalten $\mathbf{2 5}$ von ihnen 86 Prozent der derzeit entdeckten Kohlenwasserstoffe. Die restlichen 200 Becken sind praktisch noch nicht exploriert. Dazu gehören viele Offshore-Gebiete. Nach [4]

Abb. 10. a) Manganknollen sind dort angereichert, wo 1. Flïsse wenig Material vom Festland anliefern, also im Pazifischen mehr als im Atlantischen oder Indischen Ozean, 2. Strömungen - auch in der Tiefsee - das feinste Material entfernen und 3. Carbonate in großen Wassertiefen herausgelöst werden. b) Umgekehrt finden sich die größten Sedimentmächtigkeiten am Rand der Kontinente (hier Tertiär und Quartär mit über $1 \mathrm{~km}$ bis unter $0,1 \mathrm{~km}$ Dicke). Allerdings werden nur bei einer Uberdeckung von mehr als einem Kilometer Temperaturen erreicht, die zu wirtschaftlich interessanter Erdölbildung führen können (vgl. Abbildung 11). Nach [10]

Abb. 11. Schema zur Entstehung von Erdöl und Erdgas: Wenn Sedimente, die reich an organischer Substanz sind, überdeckt und dadurch höheren Temperaturen und Drüicken ausgesetzt werden, bildet sich im Bereich zwischen rund 100 und $150^{\circ} \mathrm{C}$ durch Cracken Erdöl, bei noch höheren Temperaturen Erdgas. [Nach B. P. Tissot und D. H. Welte: "Petroleum formation and occurence". Springer Verlag, Berlin - Heidelberg - New York 1978] 
10

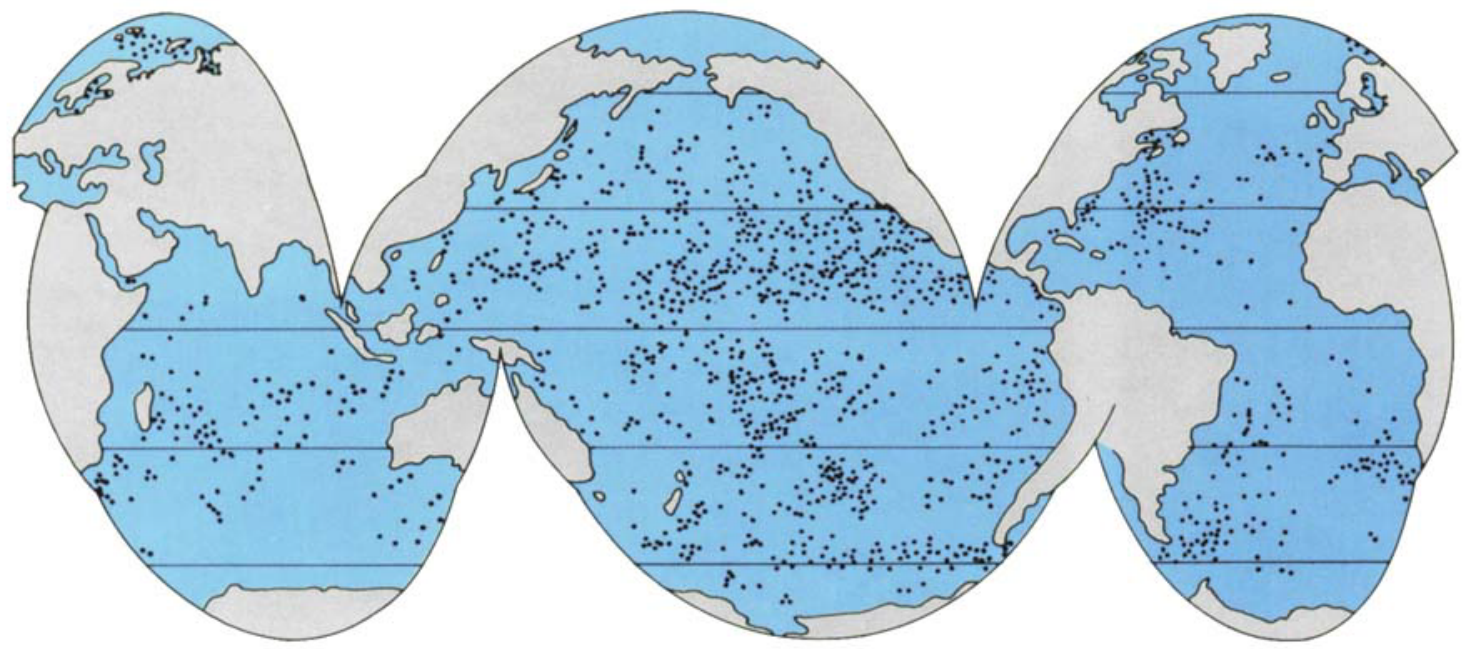

o $\quad 3000 \mathrm{~km}$

b
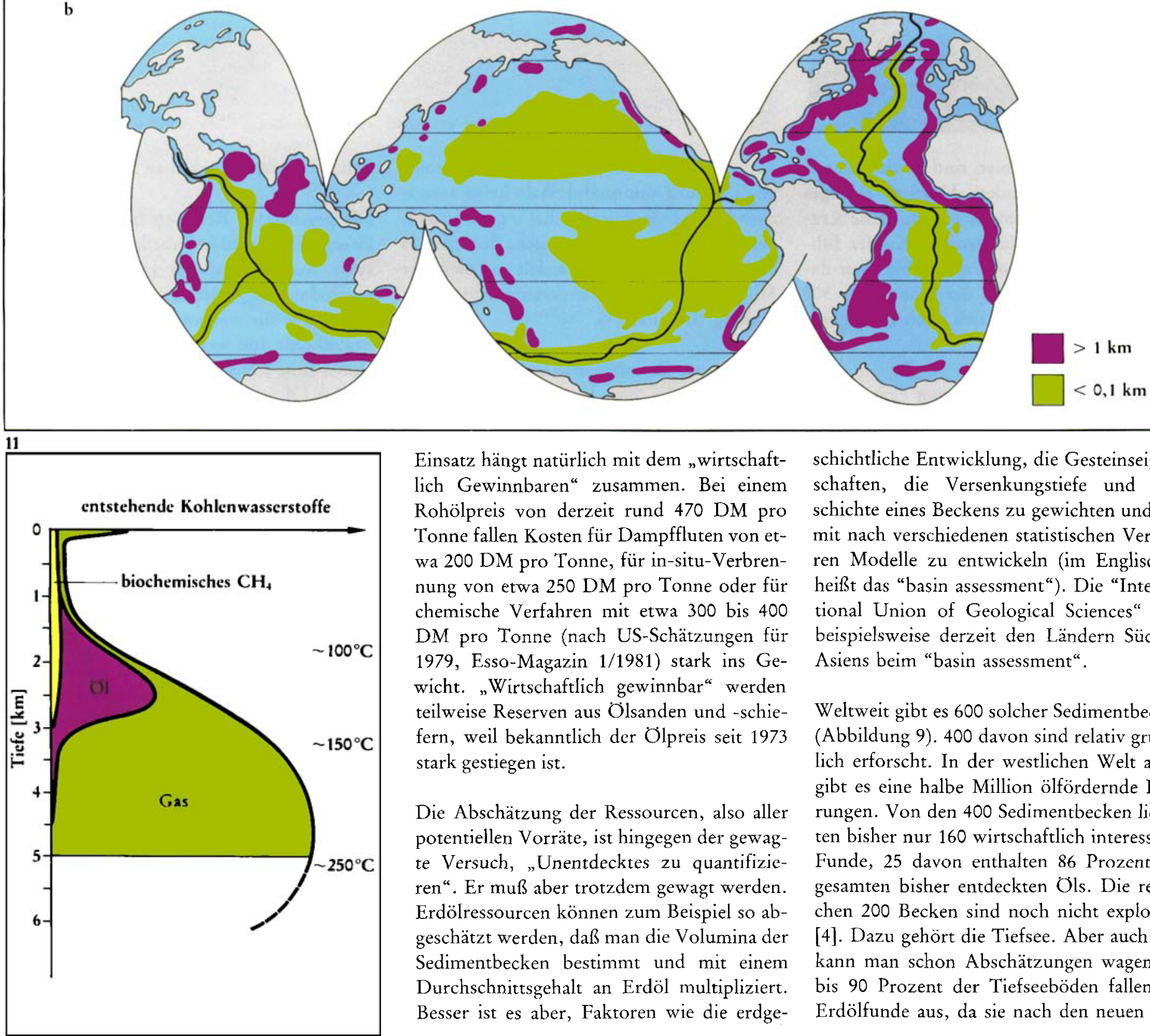

Einsatz hängt natürlich mit dem „wirtschaftlich Gewinnbaren" zusammen. Bei einem Rohölpreis von derzeit rund 470 DM pro Tonne fallen Kosten für Dampffluten von etwa 200 DM pro Tonne, für in-situ-Verbrennung von etwa $250 \mathrm{DM}$ pro Tonne oder für chemische Verfahren mit etwa 300 bis 400 DM pro Tonne (nach US-Schätzungen für 1979, Esso-Magazin 1/1981) stark ins Gewicht. "Wirtschaftlich gewinnbar" werden teilweise Reserven aus Ölsanden und -schiefern, weil bekanntlich der Ölpreis seit 1973 stark gestiegen ist.

Die Abschätzung der Ressourcen, also aller potentiellen Vorräte, ist hingegen der gewagte Versuch, "Unentdecktes zu quantifizieren“. Er muß aber trotzdem gewagt werden. Erdölressourcen können zum Beispiel so abgeschätzt werden, daß man die Volumina der Sedimentbecken bestimmt und mit einem Durchschnittsgehalt an Erdöl multipliziert. Besser ist es aber, Faktoren wie die erdge- schichtliche Entwicklung, die Gesteinseigenschaften, die Versenkungstiefe und -geschichte eines Beckens zu gewichten und damit nach verschiedenen statistischen Verfahren Modelle zu entwickeln (im Englischen heißt das "basin assessment"). Die "International Union of Geological Sciences" hilft beispielsweise derzeit den Ländern SüdostAsiens beim "basin assessment".

Weltweit gibt es 600 solcher Sedimentbecken (Abbildung 9). 400 davon sind relativ gründlich erforscht. In der westlichen Welt allein gibt es eine halbe Million ölfördernde Bohrungen. Von den 400 Sedimentbecken lieferten bisher nur 160 wirtschaftlich interessante Funde, 25 davon enthalten 86 Prozent des gesamten bisher entdeckten Öls. Die restlichen 200 Becken sind noch nicht exploriert [4]. Dazu gehört die Tiefsee. Aber auch hier kann man schon Abschätzungen wagen: 80 bis 90 Prozent der Tiefseeböden fallen für Erdölfunde aus, da sie nach den neuen $\mathrm{Hy}$ - 
12

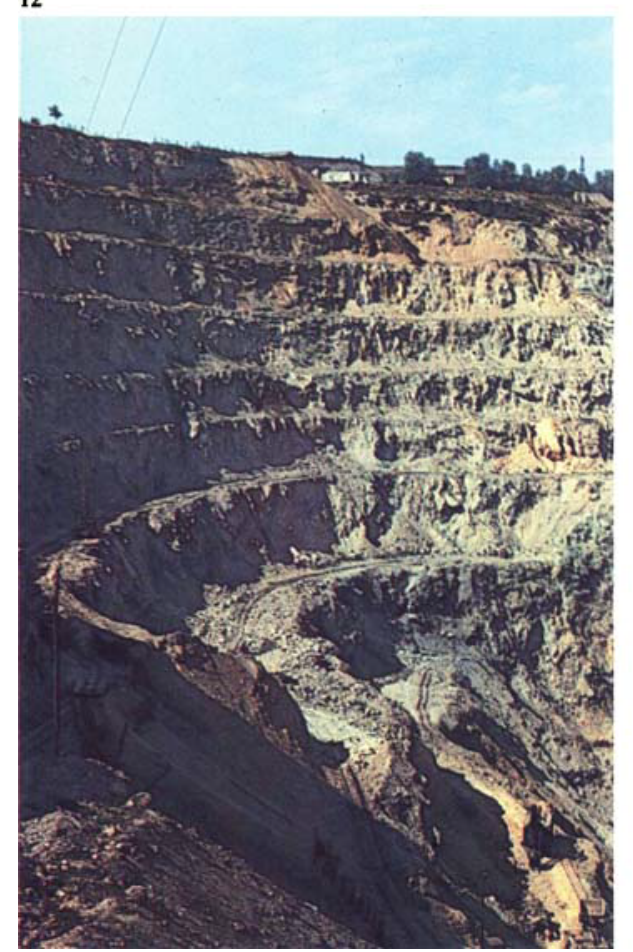

Abb. 12. In Ostserbien sind an der Wende Kreide/Tertiär Andesit-Massive hochgedrungen. Sie enthalten verschiedene Erzkörper, die bis sechs Prozent Kupfer führen. Bei Bor in Jugoslawien wird einer davon abgebaut, der an der Oberfläche 300 mal $100 \mathrm{~m}$ mißt und sich nach unten verjüngt.

\begin{abstract}
Abb. 13. Sickerwässer können Erzminerale lösen und - in der Nähe des heutigen oder früheren Grundwasserspiegels - wieder ausfällen. So wurden die ursprünglichen Eisen- und Kupfersulfide aus den obersten $50 \mathrm{~m}$ des Erzkörpers von Bor (vgl. Abbildung 12) entfernt und das Kupfer als Malachit darunter angereichert.
\end{abstract}

13

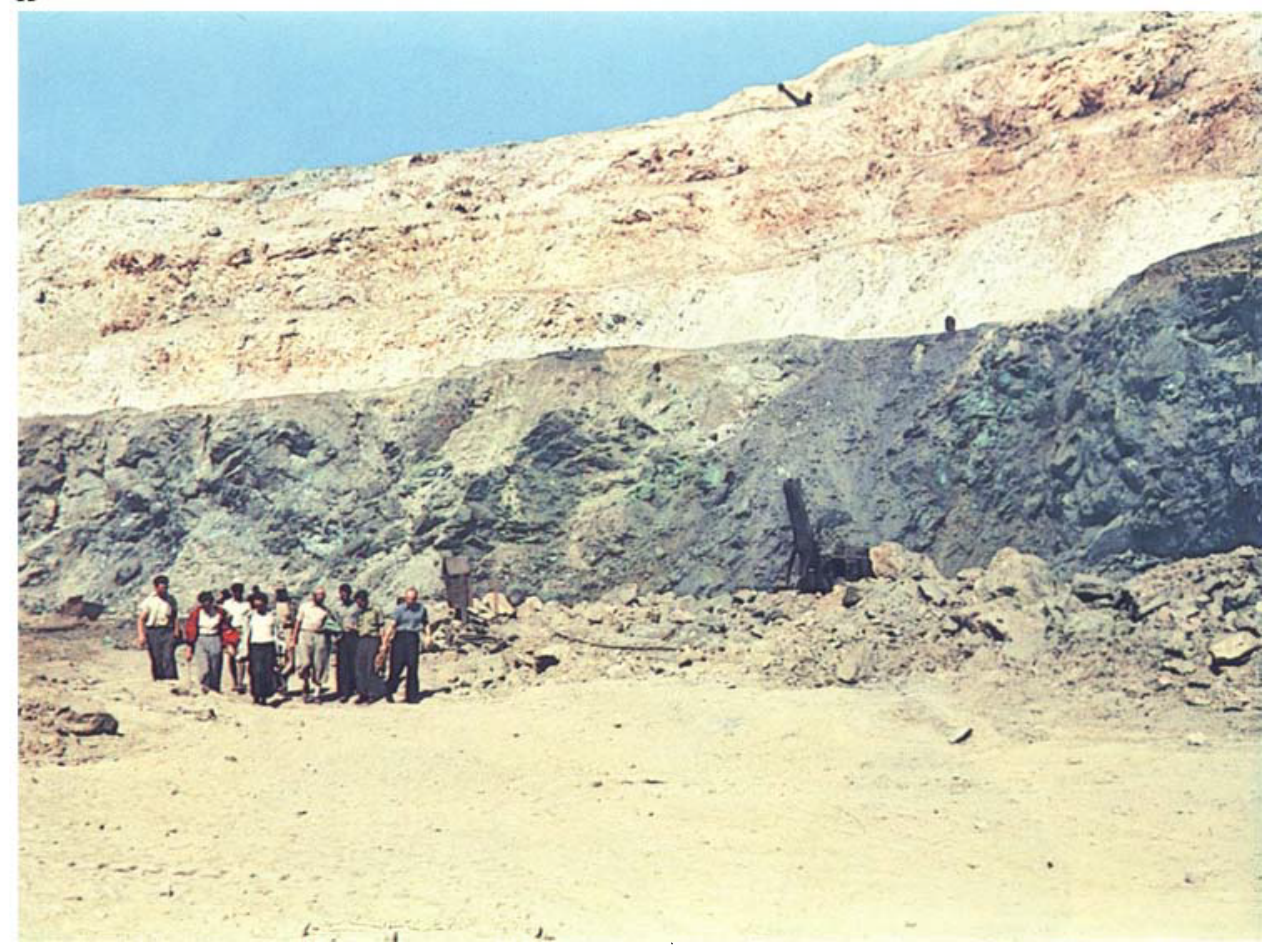

pothesen zur Entstehung der Ozeane geologisch $\mathrm{zu}$ jung sind und deshalb keine ausreichend dicke Sedimentdecke tragen, die in nennenswertem Maß organische Substanz in Kohlenwasserstoffe verwandelt und diese zur Migration in die Speichergesteine bringt (Abbildung $10 \mathrm{~b}$ und 11 ).

Die neueste Zusammenstellung der Energierohstoffvorräte hat die „Bundesanstalt für Geowissenschaften und Rohstoffe", Hannover, 1980 auf der Weltenergiekonferenz in München vorgelegt [5]. Sie basiert auf einer detaillierten Umfrage in allen Ländern, die verständlicherweise unterschiedlich entgegenkommend und zuverlässig beantwortet wurde.

Das Ergebnis zeigt, daß die Gesamtheit der fossilen Energieträger derzeit eine sichere Energiereserve von $33 \cdot 10^{21}$ Joule, d.h. etwa 1100 Milliarden Tonnen Steinkohleeinheiten (SKE*) darstellen (Tabelle 1). Fast zwei Drittel davon entfallen auf die Kohle. Der Anteil des Urans (Uran-235) von rund 1,9 $10^{21}$ Joule ist sicherlich die größte Unbekannte dabei. Deshalb wollen wir an dieser Stelle nicht näher darauf eingehen. Das spaltbare Uran-235 kann ja bekanntlich auch im Schnellen Brüter erzeugt werden. Man hofft, daß es für alle fossilen Energieträger etwa das

$* 1 \mathrm{SKE}=$ mittlerer Energieinhalt von $1 \mathrm{~kg}$ Steinkohle $=7000 \mathrm{kcal}=29288 \mathrm{~kJ}$.
Zehnfache der Reserven an Ressourcen gibt.

Die geschätzten Reserven für die einzelnen Energieträger sind in Tabelle 2 zusammengestellt. Auf der Basis der gegenwärtigen Jahresproduktion würden die Reserven an Kokskohle für weitere 100 Jahre und die an Braunkohle für weitere 130 Jahre reichen, wobei diese Rohstoffe regional gleichmäßiger als das $\mathrm{Ol}$ verteilt sind und im allgemeinen näher an den derzeitigen Verbraucherzentren liegen. Bedrohlich ist bekanntlich die Lage bei den Kohlenwasserstoffen. Die Reserven an Rohöl und Kondensaten liegen bei 95 Milliarden Tonnen. Die Reserven der konventionellen Lagerstätten würden daher bei einem derzeitigen Jahresverbauch von drei Milliarden Tonnen noch für rund 30 Jahre reichen, die Gasreserven noch weitere 50 Jahre. Es kann uns nicht trösten, daß diese "statische" Lebensdauer von 30 Jahren schon seit einem halben Jahrhundert fortgeschrieben wurde. Wenn unsere Weltwirtschaft und unser Lebensstandard weiter wachsen sollen, wenn daran zudem auch die Entwicklungsländer mit ihrem Bevölkerungszuwachs teilhaben sollen, wenn wir berücksichtigen, daß schließlich auch das letzte Aufgebot in der Tiefsee und in Polargebieten aktiviert sein wird, falls dafür genügend Kapital bereitgestellt werden kann, dann schrumpfen diese 30 Jahre rasch zusammen. Freilich, wir wissen noch nicht viel über die Ressourcen aus Olsanden und -schiefer, da sie noch nicht so intensiv exploriert worden sind. Immerhin 
Tabelle 1. Energiereserve (in $10^{21}$ Joule $)$ in fossilen Energieträgern [5].

$\begin{array}{ll}\begin{array}{l}\text { Reserven } \\ \text { (sicher gewinnbar) }\end{array} & \begin{array}{l}\text { Ressourcen } \\ \text { (zusätzlich, geschätzt) }\end{array}\end{array}$

$\begin{array}{lcc}\text { Kohle } & 20,3 & 296 \\ \text { Kohlenwasserstoffe } & 11,2 & 51 \\ \text { Uran (Anteil an Uran-235) } & 1,9 & 2,6\end{array}$

Summe

rund 33

rund 350

$* 10^{21} \mathrm{~J}=34,13 \cdot 10^{9} \mathrm{tSKE}$

Tabelle 2. Geschätzte Reserven für die einzelnen Energieträger (in 10 $0^{21}$ Joule) [5].

Reserven

$\begin{array}{lc}\text { Steinkohle } & 14,3 \\ \text { Braunkohle } & 5,85 \\ \text { Torf } & 0,17\end{array}$

$\begin{array}{ll}\text { Rohöl + Kondensat } & 4,1 \\ \text { Erdgas } & 2,8 \\ \text { Ölsande (1978) } & 1,8 \\ \text { Ólschiefer (1979) } & 1,8\end{array}$

Uran (Anteil an Uran-235) Tonnen geschätzt. Die Divergenz beruht un- sind schon jetzt vergleichbare Reserven (86 Milliarden Tonnen) nachgewiesen, deren Gewinnung freilich sehr viel kostspieliger sein wird. Die konventionellen Öl-Ressourcen werden derzeit auf 220 bis 350 Milliarden ter anderem auf unterschiedlichen Erwartungen in bezug auf die Entölungsrate. Olsande und -schiefer sollen zusätzlich noch 370 bis 700 Milliarden Tonnen an Ressourcen liefern.

Global betrachtet sieht also die Situation noch nicht beunruhigend aus [6]. Auf das dramatische regionale Ungleichgewicht von Förder- und Verbraucherländern oder auf politische Unsicherheitsfaktoren wollen wir hier nicht eingehen. Eine grobe geologische Uberschlagsrechnung macht allerdings nachdenklich: Bis 1. Januar 1979 sind rund 50 Milliarden Tonnen Erdöl gefördert worden und standen noch 90 Milliarden Tonnen als Reserven zur Verfügung. Nimmt man als Optimist noch 300 Milliarden Tonnen Ressourcen für die konventionelle Olförderung hinzu, so kommt man zu einer denkbaren Gesamtausbeute von rund 450 Milliarden Tonnen. Das meiste Ol kommt aus JuraSchichten, die 150 Millionen Jahre alt sind. Betrachtet man dieses Alter als groben Mittelwert, so hat uns die Natur in jeder Jahrmillion drei Milliarden Tonnen Erdöl zur Verfügung gestellt, also die Menge, die wir derzeit in einem Jahr verbrauchen. Wir leben also in erschreckendem Ausmaß über unsere Verhältnisse. In der Sprache eines Chemikers gilt ähnliches selbst für die Kohle: „Unsere heutige materielle Kultur ist zum erheblichen Teil auf die Ausnutzung der fossilen Brennstoffe, Steinkohle und Braunkohle, gegründet. Eine spätere Zeit wird uns aber nicht den Vorwurf ersparen, daß wir mit diesem kostbaren Material arge Verschwendung getrieben haben. "Emil Fischer sagte das im Jahre 1911 am Gründungstag der Kaiser-WilhelmGesellschaft.

Eine Abschätzung der Erzreserven ist noch schwieriger, da man im allgemeinen keine Volumenberechnungen anstellen kann. Warum? Erdöl ist an Sedimente gebunden, die so geringen Drücken und Temperaturen unterworfen waren, daß sie nicht zu metamorphen Gesteinen, etwa zu Gneisen, geworden sind. Sedimentbecken kann man deshalb einigermaßen abgrenzen und ihr Volumen bestimmen. Die wesentlichen Erzlagerstätten sind aber gerade an diese metamorphen oder magmatischen Gesteine gebunden. Dieses "Grundgebirge" setzt sich in wirtschaftlich unerreichbare Tiefen fort. Es tritt in den sogenannten stabilen Schilden zutage, in Kanada, Australien, Südafrika, Brasilien. Manche Eisenerze, der Bauxit als Aluminium-Lieferant, Mangan mit Begleitmetallen in den Manganknollen und sonst noch einiges sind allerdings Ausnahmen, da sie sedimentär entstanden sind. Eine andere grundsätzliche Schwierigkeit bei der Abschätzung der Erzvorräte ist, daß wir noch keine klaren Vorstellungen davon haben, ob der Erdmantel, aus dem die Erze letztlich stammen, primär inhomogen oder homogen ist und Metallkonzentrate durch differenzierende geochemische oder tektonische Prozesse entstanden sind. Man behilft sich daher derzeit damit, die potentiell zu gewinnenenden Ressourcen $\mathrm{R}$ (in Tonnen) aus der Krustenhäufigkeit $\mathrm{A}$ der Elemente (in Gramm pro Tonne) nach der Faustregel: $\mathrm{R}=2,45 \mathrm{~A} \cdot 10^{6} \mathrm{zu}$ berechnen. Da die Angaben für Reserven auch von der Technologie und den ständig schwankenden Metallpreisen abhängen, werden alle Angaben noch unsicherer, auch wenn man Korrekturen aus der Praxis anbringt. Auf Zahlen wollen wir daher hier verzichten, da es uns nur um das Grundsätzliche geht und es natürlich auch ganz neue Lagerstättentypen geben könnte, an die heute noch niemand denkt.

Ein Beispiel sind die Manganknollen, über die man derzeit soviel liest, die man aber als Rohstoff vor 30 Jahren noch gar nicht kannte. Sie sind im Durchschnitt kartoffelgroß und im wesentlichen in der landfernen Tief- 
see angereichert (Abbildung 10a). Da nur ihre Vorkommen an der Oberfläche des Meeresbodens für eine wirtschaftliche Gewinnung interessant sind, sollte die Menge dieses „Zweidimensionalen" Erzes leicht abzuschätzen sein. Wie war es wirklich? In den sechziger Jahren wurden optimistische Vorratsschätzungen bis zu 2000 Milliarden Tonnen publiziert - das war einer der Gründe, warum den Manganknollen auf der Seerechtskonferenz eine so große Bedeutung zugemessen wird. Noch größere Optimisten rechneten sogar aus, daß die Knollen - wie echte Kartoffeln - in dem Maße wachsen, in dem sie ausgebeutet würden. Durch genauere Untersuchungen in den siebziger Jahren ergab sich allerdings, daß Manganknollen mit höherem Gehalt (d.h. insgesamt über zwei Prozent) an den sogenannten Wertmetallen (Kupfer, Nickel, Cobalt) auf wesentlich kleinere Areale beschränkt sind, daß sie oft nur fleckenhaft vorkommen, daß morphologische Hindernisse den Abbau stören können und daß für eine wirtschaftliche Gewinnung die Felder eine Mindestgröße haben müssen. Damit schrumpfen die Reserven für die erste Generation des Tiefseebergbaus, wie er für den Nordpazifik geplant wird, auf rund 30 bis 60 Milliarden Tonnen und die Ressourcen auf rund 140 bis 200 Milliarden Tonnen. Freilich würden diese Reserven allein schon ungefähr den Nickel-Reserven an Land entsprechen und ein Vielfaches der entsprechenden Cobalt-Reserven ausmachen [7].

Noch größere Vorsicht ist geboten, wenn wir für eine Abschätzung der Erzvorkommen die Erdoberfläche verlassen. Diese hat mitunter einen besonders günstigen Einfluß auf Anreicherungsprozesse: Flüsse und das Meer reichern Seifen an; das Grundwasser laugt aus, schafft poröse Strukturen und kann durch Oxidation Ausfällungen verursachen (Abbildung 12 und 13). Der "Eiserne Hut" über Erzlagerstätten weist auch auf andere Metallanreicherungen hin, die im Mittelalter Teile Deutschlands so reich machten.

Bei all diesen Unsicherheiten zögert man mit der Aussage, daß schon bei gleichbleibendem Verbrauch, also noch nicht einmal bei einerimmerhin denkbaren - Verdoppelung bis zum Jahr 2000, viele der heute bekannten Reserven in weniger als fünfzig Jahren erschöpft sein werden, z.B. Kupfer, Blei, Zink, Zinn, Wolfram, Gold, Silber, Quecksilber und Molybdän [8]. Sicher werden zusätzliche Lagerstätten mit unseren derzeitigen Vorstellungen oder nach neuen Konzepten entdeckt.
Sicher werden eine bessere Verwertung von Schrott und sonstigen Abfällen sowie die Substitution vieler Metalle durch andere $\mathrm{Ma}$ terialien diese düsteren Prognosen widerlegen. Sicher ist aber auch, daß wir immer ärmere Erze aufarbeiten und immer entlegenere Gebiete explorieren müssen.

Für die meisten Maßnahmen zur Verlängerung der Lebensdauer dieser Vorräte ist zusätzlich Energie erforderlich. Setzt man zum Beispiel den Energieaufwand bei der Aluminiumgewinnung aus Bauxit mit rund 20 Prozent $\mathrm{Al}_{2} \mathrm{O}_{3}$ - dem derzeitigen "cut-off-grade" - gleich 1, so steigt dieser Aufwand bei zehnprozentigem Bauxit auf 1,3 und bei fünfprozentigem Bauxit auf 2,2 [8]. Oder: 1900 lag der "cut-off-grade" für Kupfererz bei drei Prozent, 1975 dagegen bei 0,35 Prozent. 1900 mußten daher zur Gewinnung von einer Tonne Kupfer rund dreißig Tonnen Gestein herausgesprengt, transportiert, gebrochen, gemahlen und aufbereitet werden; heute sind es 300 Tonnen, die außerdem wieder auf Halde geschafft werden müssen.

\section{Aussichten}

Was ist also aus geologischer Sicht zu tun? Erstens müssen die Prospektions- und Explorationsmethoden verbessert werden, denn wir brauchen Neufunde von Rohstoffen z.B. in größerer Tiefe oder in noch unbekannten Gebieten wie unter den Meeren, unter Eis, unter Urwald oder unter Sedimentdecken. Als Hilfsmittel braucht man dafür geologische Karten auf dem -300- oder - $600 \mathrm{~m}$ Niveau. Wir müssen uns um neue Konzepte bemühen und sie in der Praxis testen, wie das mit den Theorien zur Entstehung der Ozeane im letzten Jahrzehnt so erfolgreich mit Hilfe der Tiefseebohrungen demonstriert worden ist. Die Kontinente werden für uns jedoch immer eine größere Bedeutung haben als die Meere. Auch neue Theorien zur Entstehung von Lagerstätten müssen durch Bohrungen gesichert werden, vor allem durch Tiefstbohrungen. Der Geologe muß zweitens immer wieder darauf hinweisen, daß unsere Erde trotz einfallender Meteorite und ausreißender Raumsonden - stofflich ein geschlossenes System ist. Das bedeutet, daß die Entropie nicht nur hinsichtlich der Energie, sondern auch hinsichtlich der Materie zunimmt, was eindrucksvoll durch die Umwandlung von $\mathrm{O} 1$ oder Kohle in $\mathrm{CO}_{2}$ bei der Verbrennung demonstriert wird. Der Abbau der Materie, über die wir verfügen können, in Materie, die unserer Nutzung verlorengeht, wird vom
Menschen beschleunigt [9], auch wenn ihre Gesamtmenge gleichbleibt und sich die geochemischen Kreisläufe schließen - allerdings oft im für uns unerreichbaren Ozean und in für uns unerreichbaren Zeiträumen.

Wir beginnen, mit der Wiederverwendung ("recycling") die Pflanzen nachzuahmen, auch wenn die Herbstblätter am Boden der Laubwälder immer noch schöner sind als eine Mülldeponie, in der wir zunehmend auf Rohstoffsuche gehen müssen. Müll ist derzeit ja weithin noch nicht Abfall wie im Wald, sondern nur Weggeworfenes, Symbol einer Wegwerfgesellschaft für Landschaften, Pflanzen- und Tierarten, im letzten Sinn also auch für das Wegwerfen von Tradition.

Wir werden immer mehr substituieren müssen. Materiell durch den künftigen Ersatz von Seltenerem durch Häufigeres, von Rohstoffen, deren Gewinnung und Verarbeitung viel Energie erfordern, durch solche, bei denen wir sparen können. Vielleicht fallen wir wieder in die Eisenzeit zurück. Die funktionelle Substitution ist in vollem Gang und läßt sich gerade umgekehrt mit dem Schlagwort "From steel and ships to genes and chips" charakterisieren. Sie fordert ja das Seltene, z.B. Yttrium, Indium, Gallium, Germanium oder Elemente der Platin-Gruppe. Das Hauptproblem auf dem Weg von der Suche nach Rohstoffen bis zur Gewinnung, Verarbeitung und schließlich Minimierung der Umweltbelastung bleibt die Beschaffung ausreichender Energie zu realistischen Kosten und die Abwägung der vielen Faktoren und Probleme bei den unterschiedlichen Energiequellen.

Wir alle müssen einen Weg „zwischen Endzeitpanik und gottvertrauender Gelassenheit" (W. Casper, 1974) finden. Aus geologischer Sicht kommt man zu dem Schluß, daß auf längere Sicht kein absoluter Energiemangel auftreten wird, daß uns aber auch bei politischer Stabilität kurzfristige Engpässe drohen, bis bekannte, verbesserte und neue Technologien einschließlich der Verwendung von Kernenergie wirksam helfen können. Bei der Energieversorgung auf sehr lange Sicht sollten wir uns daran erinnern, daß die Sonne in zwei Wochen der äußeren Erdatmosphäre so viel Energie zuführt, wie alle fossilen Brennstoffvorräte zusammen schließlich liefern können. Warum also soll der Jäger nach Olfeldern nicht in vielen Bereichen zum Sammler, ja zum Ackerbauer von Sonnenenergie werden? Diese Ressource hat freilich 
ihre spezifischen physikalischen, technologischen und wirtschaftlichen Probleme. Sie ist aber so unerschöpflich wie die Ressource neuer wissenschaftlicher und technischer Ideen.

Und schließlich: Wir Menschen haben auch noch andere ureigene Reserven einzusetzen. Wir leben nicht von Rohstoffen und Brot allein, was materielle Einschränkungen in den Industriestaaten erleichtern wird, in dem Drittel der Welt also, das derzeit 90 Prozent ihrer Rohstoffe verbraucht. Wir können aber auch weltweit nicht auf Rohstoffe und Brot verzichten, was Einschränkungen im Bevölkerungswachstum der anderen zwei Drittel exzwingen wird. Es ist immer noch das vernünftigste, lieber künftige Reue zu minimieren, als die Erfüllung heutiger Ansprüche zu maximieren. Was aber in den kommenden Jahren und Jahrzehnten wirklich drängt, ist die Zeit: "Jetzt ist die Zeit für uns zu begreifen, daß wir unsere natürlichen Rohstoffvorräte nicht leichtfertig vergeuden dürfen. Eine Verschwendung jedoch, die am wenigsten wiedergutzumachen ist, ist die Verschwendung von Zeit." [R. S. McNamara in einer Rede am 2. Oktober 1979 in Belgrad]

\section{Literatur}

[1] E. Cook: "Limits of Exploitation of Nonrenewable Resources". Science 191, 677 (1976).

[2] E. Seibold: „Rohstoffe in der Tiefsee Geologische Aspekte“. Rheinisch-Westfälische Akademie der Wissenschaften. Vorträge N 283, S. 49. Düsseldorf 1979.

[3] D. A. Brobst und W. P. Pratt (Hrsg.): "United States Mineral Resources". Geological Survey Prof. Paper 820. Washington DC 1975.

[4] E. E. Hotz: The Future of Hydrocarbon Exploration. In: F. Bender (Hrsg.): The Mineral Resource Potential of the Earth. Proc. 2. Int. Symp. Hannover 1979. S. 100.

[5] Bundesanstalt für Geowissenschaften und Rohstoffe: "Survey of Energy Resources 1980". 11. Weltenergiekonferenz in München. London 1980.

[6] R. Gerwin: „Die Welt-Energie-Perspektive“. Deutsche Verlags-Anstalt, Stuttgart 1980.
[7] E. Seibold: "Deep Sea Manganese Nodules - The Challenge since 'Challenger' ". Episodes $1978,4,3$.

[8] P. Cloud: "Entropy, materials and posterity“. Geologische Rundschau 66, 3, 678 (1977).

[9] Science Council of Canada: "Entropy and the economic process: A Seminar". Ottawa 1980.

[10] E. Seibold und W. H. Berger: "The Sea Floor - An Introduction to Marine Geology“. Springer-Verlag, Berlin - Heidelberg New York 1982.

Zusätzliche allgemeine Hinweise finden sich in:

W. Gocht: "Wirtschaftsgeologie“. SpringerVerlag, Berlin-Heidelberg-New York 1978.

C. U. Sames: „Die Zukunft der Metalle“. Suhrkamp Taschenbuch 157. Frankfurt a.M. 1974.

Prof. Dr. E. Seibold, geb. 1918 in Stuttgart, studierte vor dem Krieg in Tübingen Naturwissenschaften, danach in Bonn und Tübingen Geologie, wo er 1948 promovierte und sich 1951 habilitierte. Nach einer Dozentur an der Technischen Hochschule in Karlsruhe wurde er 1954 Extraordinarius am Geologisch-Paläontologischen Institut der Universität Tübingen. 1958 wurde er als Direktor des Geologisch-Paläontologischen Instituts an die Universität Kiel berufen. Er blieb ihr seitdem trotz verschiedener Rufe verbunden. Seit 1980 ist er Präsident der Deutschen Forschungsgemeinschaft und Präsident der Internationalen Union Geologischer Wissenschaften. Sein hauptsächliches Arbeitsgebiet ist die Meeresgeologie. Er war mehrfach Fahrtleiter auf verschiedenen Forschungsschiffen. Die Ergebnisse der Expeditionen schlugen sich in über 130 eigenen Veröffentlichungen und rund 40 Dissertationen nieder. - Der Autor dankt der Bundesanstalt für Geowissenschaften und Rohstoffe in Hannover und den Geological Surveys Kanadas in Ottawa, Südafrikas in Pretoria und der USA in Washington, DC, für viele Anregungen. 